\title{
Study on awareness, perceptions, experiences in decision process of hysterectomy in women presenting to Goa Medical College
}

\author{
Manjusha Jindal*, Viraj Ramesh Naik, Mrinalini Sahasrabhojanee, Jagadish Cacodcar, \\ Manojkumar S. Kulkarni, Smita Naik
}

Department of Obstetrics and Gynaecology, Goa Medical College, Bambolim, Goa, India

Received: 08 January 2021

Accepted: 10 February 2021

\section{*Correspondence:}

Dr. Manjusha Jindal,

E-mail: manjushajindal@gmail.com

Copyright: (c) the author(s), publisher and licensee Medip Academy. This is an open-access article distributed under the terms of the Creative Commons Attribution Non-Commercial License, which permits unrestricted non-commercial use, distribution, and reproduction in any medium, provided the original work is properly cited.

\begin{abstract}
Background: Hysterectomy (removal of uterus) has many indications like fibroids, dysfunctional uterine bleeding, uterine prolapse and chronic pelvic pain. The purpose of this study is to know the level of awareness of women about hysterectomy, to study their experiences, to assess their involvement in decision making process and to develop a suitable protocol, counselling and education material to ensure women's involvement.

Methods: This is a cross sectional study using validated semi-structured interview questionnaire over 6 months (1st December, 2018 to 31st May, 2019) in Goa Medical College. Data was entered in epidata manager and analysed using SPSS 22 version. Categorical variables were expressed in percentages and proportions and quantitative variables in mean \pm SD. Significance was checked using Chi square test and association between dependent and independent variables was assessed by using bivariate analysis. P value $<0.05$ was taken as significant.

Results: A total of 149 women were recruited into the study who were admitted for hysterectomy. The mean age of women was 49.3 years. The awareness with respect to various aspects of hysterectomy was good in $23.4 \%$ subjects and poor in $76.6 \%$ subjects. The awareness was significantly related to level of education $(\mathrm{p}=0.002)$, urban location $(\mathrm{p}=0.016)$ and socioeconomic status $(\mathrm{p}=0.028)$. Education was significant factor in decision-making process $(\mathrm{p}<$ 0.05). It was observed that good experience had positive correlation to their perception about hysterectomy.

Conclusions: It was concluded that awareness about hysterectomy was significantly related to education, urban location and socio-economic status of women. Pre-operative education would help to reduce the post-operative symptoms for a women undergoing hysterectomy.
\end{abstract}

Keywords: Awareness, Attitudes, Experience, Hysterectomy, Knowledge, Perception

\section{INTRODUCTION}

Hysterectomy, the removal of the uterus, is the leading reason for non-obstetric surgery in women in various income settings. ${ }^{1,2}$ There are many medical indications for hysterectomy, which include fibroids, dysfunctional uterine bleeding, uterine prolapse and chronic pelvic pain. ${ }^{3-5}$

All over the World, hysterectomy is the most common surgery in women next only to Caesarean section. There is a huge variation in the rate of hysterectomy in various parts of the world ${ }^{6}$ Hysterectomy prevalence was found to be $0.2-6.3$ per 100 women in age group $15-49$ years for 21 out of 36 states and Union Territories of India as per study done by Prusty et al. ${ }^{7}$ The importance of providing detailed information about treatment options to patients and also participation of patients in treatment decision making is being stressed upon by the governmental, health professional and health care provider organisations. ${ }^{8}$ Hysterectomy is associated with various psychosocial effects in context to pain experienced, sexual functioning and pre-operative as well as postoperative anxiety. Operative procedure can be physically 
and psychologically stressful for patients. There are many aspects of this surgery, which affect patient's health. It is observed that most of these aspects are related to the patient's knowledge about the surgery and its outcomes.

The psychological, physical and sexual effects of hysterectomy are conflicting and the findings are found to be mixed. ${ }^{9}$ It is established that side-effects of hysterectomy incorporate both positive and negative physical or emotional aspects, and there have been no studies examining these effects among Indian rural women. $^{2}$

While some studies showed that patients who underwent hysterectomy have experienced greater improvement in their mental health, sexual desire and functioning, and overall satisfaction, while others show that patients report many negative outcomes, with ill effects on sexual functioning being the main concern. ${ }^{2}$ There are several studies in western countries which explored women's choice to undergo hysterectomy versus non-surgical approaches for benign conditions and few studies also explored women's decision making relating to various surgical approaches available for hysterectomy. ${ }^{10,11}$

The knowledge with regards to the surgery in patients is very low, especially in developing country like India. Elgi MC et al in their study showed that the educational intervention is effective in reducing the anxiety and pain among experimental group. ${ }^{12}$ The present study is proposed as there is limited evidence in published medical literature regarding the same and to our knowledge no such study has been done in the state of Goa.

Objectives of the study were to study the level of awareness regarding hysterectomy among women at the tertiary care hospital in Goa. To study the perceptions, choices and expectations towards undergoing hysterectomy. To analyse their experiences while undergoing hysterectomy. To assess their involvement in decision making process of hysterectomy. To develop suitable protocols, counselling and education material to ensure women's improved involvement in decision making process.

\section{METHODS}

The study was conducted at Goa Medical College Bambolim, a tertiary care hospital in Goa after approval from Institutional Ethics Committee (IEC). It was a cross sectional study using validated semi-structured interview questionnaire from 1st December 2018 to 31st May 2019. Census method was used for sample selection.

\section{Study participants}

Patients admitted in ward for hysterectomy after a careful explanation of the objectives of the study were voluntarily recruited into the study and their consent duly obtained. The interview questionnaire was administered to study participants before the surgery and their experience was recorded after the surgery.

\section{Inclusion criteria}

Study subjects were women admitted for hysterectomy (various benign indications) and willing to participate in the study.

\section{Exclusion criteria}

Women unwilling to participate in the study and undergoing hysterectomy for malignant conditions

\section{Data collection and analysis}

The source of data was the semi-structured questionnaire form filled after interview. These interviews included demographic characteristics, their awareness regarding hysterectomy procedure, involvement in decisionmaking, perceptions, attitude, expectations and experience during hospital stay. The confidentiality was maintained.

Data was entered in epidata manager and analysed using SPSS 22 version. Categorical variables were expressed in percentages and proportions and quantitative variables in mean \pm SD. Significance was checked using chi square test and association between dependent and independent variables was assessed by using bivariate analysis. $\mathrm{P}$ value $<0.05$ was taken as significant.

\section{RESULTS}

A total of 149 women were recruited into the study who were admitted for hysterectomy. The mean age of women was 49.3 years with $46.3 \%$ subjects in $40-50$ years age group and $35.6 \%$ in more than 50 years age group. $60.4 \%$ subjects had not attained menopause with mean age of 45.42 years while $39.4 \%$ were menopausal with mean age of 55.24 years. $36.2 \%$ participants were educated up to secondary level while $45 \%$ received education till primary level only. $12.8 \%$ subjects were educated up to higher secondary and above. $70.5 \%$ of study participants were residing in the rural area and remaining $29.5 \%$ were residents of urban areas. $47 \%$ participants belonged to class V, $27.5 \%$ to class IV, $17.3 \%$ to class III, and only $6 \%$ and $2 \%$ to class II and Class I respectively as per modified B G Prasad classification (Table 1).

\section{Awareness}

$95.35 \%$ subjects were aware of meaning of hysterectomy while only $23.55 \%$ were aware about different types of hysterectomy. $87 \%$ women knew abdominal hysterectomy as the route of hysterectomy. Less invasive routes like vaginal hysterectomy, laparoscopic and robotic hysterectomy; were known to $46.3 \%, 12.1 \%$ and $2.7 \%$ study subjects only. $129(86.6 \%)$ participants were 
aware about alternate treatment options and almost all $144(96.6 \%)$ women undergoing hysterectomy agreed that operation was indicated and were aware of complications related to surgery.

Table 1: Socio-demographic characteristics of the study participants.

\begin{tabular}{|ll|}
\hline Characteristic & $\begin{array}{l}\text { Total number (\%) } \\
\mathbf{n}=\mathbf{1 4 9}(\%)\end{array}$ \\
\hline Age in years & \\
\hline$<40$ & $27(18.1)$ \\
\hline $40-50$ & $69(46.3)$ \\
\hline$>50$ & $53(35.6)$ \\
\hline Attained menopause & $59(39.6)$ \\
\hline Yes & $90(60.4)$ \\
\hline No & \\
\hline Education & $09(6)$ \\
\hline Illiterate & $67(45)$ \\
\hline Primary & $54(36.2)$ \\
\hline Secondary & $15(10.1)$ \\
\hline Higher secondary & $03(2)$ \\
\hline Graduate & $01(0.7)$ \\
\hline PG & \\
\hline Location & $44(29.5)$ \\
\hline Urban & $105(70.5)$ \\
\hline Rural & \\
\hline Socioeconomic status (according to modified BG \\
Prasad classification) & $03(2)$ \\
\hline I & $09(6)$ \\
\hline II & $26(17.4)$ \\
\hline III & $41(27.5)$ \\
\hline IV & \\
\hline V & \\
\hline
\end{tabular}

Only one third $(32.8 \%)$ of the respondents were aware of possibility of preservation and removal of ovaries and $28.2 \%$ knew about benefits of preservation and consequences of removal of ovaries and requirement of hormone replacement therapy after ovaries were removed. Only 39 women $(26.2 \%)$ were aware about the different incisions on abdomen given during surgery. $89.3 \%$ subjects knew the choice of anaesthesia but the awareness about anaesthesia procedure and complications was known to only $19.5 \%$ subjects. Thus the awareness with respect to hysterectomy was good in $23.4 \%$ subjects and poor in $76.6 \%$ subjects. The awareness was significantly related to level of education $(\mathrm{p}=.002)$, urban location $(\mathrm{p}=.016)$ and socioeconomic status $(\mathrm{p}=.028)$.

\section{Choice and involvement in decision making}

A total $43.6 \%$ women were involved in decision making process depending on awareness about route of hysterectomy, indication of hysterectomy, alternate treatment options, complications and care associated with operation, and preservation/removal of ovaries.
Education was significant factor $(p=.010)$ in their decision making choices.

\section{Attitude, perception and expectation}

$16.8 \%$ study subjects were concerned about the surgery as they didn't understand the complications of surgery $(3.4 \%)$, post operative care and need to follow up (3.4\%), and post operative precautions $(26.7 \%) .83 .2 \%$ subjects had positive attitude towards the surgery and it was significantly related to their awareness $(\mathrm{p}=.003) .27 .5 \%$ perceived hysterectomy as loss of feminity. $24.8 \%$ responders felt that they would be sad after hysterectomy. Sadness was attributed to major surgery event $(16.8 \%)$ or loss of womb (55.7\%). 41.6\% participants felt that they will not be able to continue sexual activity after hysterectomy. Majority of study subjects $(92.8 \%)$ felt that they would feel better after interacting with women who underwent similar surgery. $87.6 \%$ felt that an information leaflet would help them in decision-making.

\section{Experience}

A total $95.3 \%$ subjects had good experience during their hospital stay. $97.3 \%$ responders felt that Doctors were empathetic, while $94 \%$ and $89.9 \%$ of responders felt anaesthetist and nurses to be empathetic. Good experience had positive correlation to their perception about hysterectomy $(\mathrm{p}=.000)$.

\section{DISCUSSION}

In the present study, mean age of the study subjects was 49.3 years. The findings are similar to findings reported by Prusty et al, wherein among all planned hysterectomy cases, most patients were 45 years or above. ${ }^{7}$ In our study, more than half of the study subjects belonged to rural areas similar to studies done by Desai $\mathrm{S}$. et al and Shekhar et al. ${ }^{3,13}$

Nearly half of the study subjects $(47 \%)$ belonged to socioeconomic status Class V, one third (27.5\%) belonged to class IV, rest $25.5 \%$ belonged to class I, II, III. The findings of our study are similar to those of the study done by Shekhar et al. ${ }^{13}$

Most of the study subjects $(95.35 \%)$ were aware of the meaning of the term hysterectomy, less than a quarter $(23.55 \%)$ were aware of different types of hysterectomy. More than three fourth of the study subjects $(87 \%)$ were aware of abdominal hysterectomy as route of hysterectomy. Nearly half $(46.3 \%)$ of the study subjects were aware of vaginal route, $12.1 \%$ were aware of laparoscopic route and $2.7 \%$ were aware of robotic surgery.

These findings are consistent with the findings of the study done by Uskul et al, in which they interviewed 29 women who had undergone hysterectomy regarding their experiences with gynaecological complaints and how 
they decided to undergo hysterectomy. ${ }^{14}$ The authors found that majority of the women were aware about hysterectomy before they visited gynaecologist.

Also, they reported that among the women, who were interviewed, those women who knew about the alternative treatment options were the ones who already had found information themselves before consulting the gynaecologist and these women were also aware about the different routes of hysterectomy.

In our study majority of study subjects (86.6\%) were aware about alternate treatment options which is comparable to findings of study done by Skea et al wherein they found that $74.74 \%$ of the study subjects were aware of alternate treatment options. Majority of the study subjects $(96.6 \%)$, agreed that operation was indicated similar to findings by Jain et al, who reported that $98 \%$ women agreed that surgery was indicated. ${ }^{2,8}$

One third $(32.8 \%)$ of the study subjects were aware of preservation and removal of ovaries, however only $28.2 \%$ of the study subjects were aware of benefits and consequences of removal of ovaries and also requirement of Hormone Replacement Therapy. The findings correlated to findings in study done by Jain $\mathrm{S}$. et al that is $32 \%$ study subjects were aware of the requirement of Hormone Replacement Therapy ${ }^{2}$

In study done by Uskul et al, only $6.8 \%$ of the women were aware of the benefits and consequences of removal of ovaries. As most of the study subjects belonged to rural areas they did not have much concern about the oophorectomy being done. ${ }^{14}$ This observation can be attributed to their lower educational levels. In a study done by Prusty et al, women with higher educational level were less likely to opt for hysterectomy than those women who had no education or those who did study only up to primary level (OR 0.47 ; CI $0.42-0.50){ }^{7}$

Nearly a quarter of the study subjects (26.2\%) were aware of different types of incision. Around $89.3 \%$ of the study subjects were aware of the choice of anaesthesia, however less than a quarter $(19.5 \%)$ of the study subjects were aware of anaesthesia procedure and complications. Poor knowledge of study participants again can be attributed to the rural location of most of the study subjects, their socio economic status and their level of education.

The awareness with respect to hysterectomy was good in $23.4 \%$ subjects and poor in $76.6 \%$ subjects. The awareness was significantly related to level of education $(\mathrm{p}=.002)$, urban location $(\mathrm{p}=.016)$ and socioeconomic status $(\mathrm{p}=.028)$. The findings were similar to study done by Elgi et al, Shekhar et al and Dessai $\mathrm{S}$ et al. ${ }^{3,12,13}$ In all these studies there was significant association between the awareness of women about hysterectomy and their education level, locality and socio-economic status.
Nearly half of the study subjects were involved in the decision making process depending on awareness about route of hysterectomy, indications of hysterectomy, alternate treatment options, complications and care associated with surgery, preservation or removal of ovaries. Education was significant factor in decisionmaking process $(\mathrm{p}<0.05)$ in present study.

The findings of our study are similar to the findings reported by Uskul et al and Janda et al, Uskul et al, reported that majority of women were aware of hysterectomy before consulting gynaecologist and also about different routes of hysterectomy. ${ }^{8,14,15}$ In study done by Janda M. et al, $96 \%$ of the study subjects were aware about hysterectomy and possible surgical approaches. ${ }^{15}$

$16.8 \%$ of the study subjects were concerned about the surgery, as they did not understand the complications of surgery $(3.4 \%)$, post-operative care and need to follow up (3.4\%) and post-operative precautions. This can be attributed to the inadequate knowledge of the study subjects about hysterectomy. Elgi et al, studied the effect of Self Instructional Module (SIM) on the post operative problems faced by women undergoing hysterectomy.

The severity of anxiety, pain, insomnia, abdominal discomfort and fatigue was less in experimental group (those who were given SIM) than the control group. The mean score of the entire outcome was also significantly lower than in the control group. ${ }^{12}$ The findings of Elgi et al, also show that the educational intervention is effective in reducing the anxiety and other post operative symptoms among experimental group. The mean anxiety score of experimental group was $(1.8 \pm 1.88) .{ }^{12}$

Kiyohasa conducted a study to evaluate anxiety levels related to the surgery; on the day before Surgery and found that there is lower anxiety level among patients who knew about the surgery $(\mathrm{p}=0.038) .{ }^{16}$ They concluded that increased knowledge of patients regarding the surgery reduces their anxiety level. Young women who undergo hysterectomy post sterilization tend to have 'hysterectomy regret', with potentially negative impact on their mental wellbeing and quality of life as reported by Prusty et al. ${ }^{7}$

Majority of the study subjects $(83.2 \%)$, had positive attitude towards surgery. This was significantly related to awareness. These findings are consistent with findings of Uskul et al and Skea et al. ${ }^{8,14}$ In study by Uskul et al, most of the women had positive attitude towards hysterectomy as they had already consulted private physicians before consulting gynaecologist. ${ }^{14}$ Similarly, in study done by Skea et al, $90 \%$ of the study subjects were sure that hysterectomy was best treatment for them. $83.3 \%$ were of the opinion that the decision was consistent with their personal values. ${ }^{8} 95 \%$ were satisfied with their decision. This all indicated positive attitude towards hysterectomy of the study subjects. 
Nearly a quarter of the study subjects (27\%) perceived hysterectomy as loss of feminity. Also nearly a quarter of the study subjects $(24.8 \%)$ felt that they would be sad after hysterectomy, sadness being attributed to major surgical event $(16.8 \%)$ or loss of womb $(55.7 \%)$. Nearly half of the study subjects $(41.6 \%)$ were of the opinion that they would not be able to continue sexual activity.

The results of our study are similar to that reported by Jain S. et al wherein they found that $20.83 \%$ of the study subjects felt hysterectomy means loss of feminity, $39.58 \%$ of the study subjects felt that they would be sad after hysterectomy. ${ }^{2}$ This was mainly because of poor knowledge of the study subjects. The result of simple hysterectomy on women's sexuality is very complex and it involves various interpersonal factors, cultural beliefs, psychological behaviour and socioeconomic status. ${ }^{2,17}$

Majority of the study subjects $(92.8 \%)$ felt better after communicating with women who underwent similar surgery. Also majority of women $(87.6 \%)$ were of the opinion that information leaflet would help in decisionmaking process. Jain s. et al and Prusty et al in their studies also found similar findings. ${ }^{2,7}$ This again reflects the importance of preoperative knowledge prior to surgery. In study done by Skea et al, it was proposed that provision of a dedicated decision-making counselling session after women have been given information about their diagnosis and treatment options reduced the proportion of women opting for hysterectomy and improved their long term satisfaction with the decision. ${ }^{8}$

In study conducted by Kumari VJ et al, knowledge of pre and post-operative self-care management among women who were undergoing abdominal hysterectomy was assessed by a structured knowledge questionnaire using structured interview schedule. ${ }^{18}$ The result showed that, in pre- test, $56 \%$ of subjects had average knowledge and $44 \%$ of subjects had poor knowledge; however during post-test $52 \%$ of subjects had good knowledge, $42 \%$ of subjects had excellent knowledge, $6 \%$ of subjects had average knowledge and none of subjects had poor and very poor knowledge.

Hence they concluded that the structured teaching programme on pre and postoperative self-care management for women undergoing hysterectomy is effective. An experimental study conducted by Sharmila R. et al, on the effectiveness of a structured teaching programme about the knowledge regarding postoperative care concluded that the intervention was effective in improving the knowledge with regards to postoperative care. ${ }^{19}$ The mean pre-test score was 19.8 and the post test score was 26.4 and difference in mean was significant at 0.05 level.

Majority of the study subjects (95.3\%), had good experience during the hospital stay. $97.3 \%$ study subjects felt that doctors were empathetic while $94 \%$ and $89.9 \%$ of the study subjects felt anaesthetist and nurses were empathetic respectively. It was observed that good experience had positive correlation to their perception about hysterectomy.

Findings of our study are similar to the findings reported by Skea et al, Janda et al and Schoenfelder et al. ${ }^{8,15,20}$ In study done by Skea et al, $93 \%$ of the study subjects felt supported and reassured by doctors. In study done by Schoenfelder and colleagues and Janda et al on patient's satisfaction following surgery, it was found that the predictors like individualised care, clinical outcome and organisation of discharge are predictors of overall satisfaction. ${ }^{15,20}$

\section{CONCLUSION}

We conclude from our study that, majority of women were aware about hysterectomy however very few were aware of the different routes of hysterectomy. Awareness was significantly related to education, urban location and socio-economic status. Education was significant factor in decision-making process. The response was affected by their pre operative knowledge about the procedure. Efforts to improve decision-making processes for patients should address both the communication approaches of individual doctors and the framework of consultation systems within which these decisions are made. Preoperative education would help to reduce the postoperative symptoms for women undergoing hysterectomy.

\section{ACKNOWLEDGMENTS}

Authors would like to thank Prof and Head department of OBG for allowing us to conduct the study..

Funding: No funding sources

Conflict of interest: None declared

Ethical approval: The study was approved by the Institutional Ethics Committee

\section{REFERENCES}

1. Hammer A, Rositch AF, Kahlert J, Gravitt PE, Blaakaer J, Søgaard M. Global epidemiology of hysterectomy: Possible impact on gynecological cancer rates. Am J Obstet Gynecol. 2015;213(1):239.

2. Jain S, Pansare SS. Perceptions of Indian women on hysterectomy. Int J Reprod Contracept Obstet Gynecol. 2017;6:4646-51.

3. Desai S, Campbell OMR, Sinha T, Mahal A, Cousens $S$. Incidence and determinants of hysterectomy in a low-income setting in Gujarat, India. Health Policy and Planning. 2017;32(1):68-78.

4. Radha K, Devi GP, Chandrasekharan PA, Swathi P, Radha G. Epidemiology of hysterectomy-a cross sectional study among Piligrims of Tirumala. IOSR J Dent Med Sci (IOSR-JDMS). 2015;14:1-5. 
5. Verma D, Singh P, Kulshrestha R. Analysis of histopathological examination of the hysterectomy specimens in a north Indian teaching institute. Int $\mathbf{J}$ Res Med Sci. 2016;4(11):4753-8.

6. Bala R, Devi KP, Singh CM. Trend of hysterectomy: A retrospective analysis in Regional Institute of Medical Sciences (RIMS). J Med Soc. 2015;29:4-7.

7. Prusty RK, Choithani C, Gupta SD. Predictors of hysterectomy among married women 15-49 years in India. Reprod Health. 2018;15:3.

8. Skea Z, Harry V, Bhattacharya S, Entwistle V, Williams B, Maclennan G, Templeton A. Women's perceptions of decision-making about hysterectomy. BJOG. 2004;111(2):133-42.

9. Singh A, Arora AK. Why hysterectomy rates are lower in India. Ind $\mathbf{J}$ Community Med. 2008;33(3):196-7.

10. Mthiyane N. Uterinne health and women's participation in decisions to undergo hysterectomy in KwaZulu-Natal, Agenda .2012;26:80-92.

11. Womg.SK, Jakus-Waldman, Yazdany T. Patient beliefs regarding hysterectomy in women seeking surgery from pelvic organ prolapsed: Finding in a predominantly Hispanic population. Female Pelvic Med. Reconstr. Surg. 2014;20:267-271.

12. Elgi MC, Lekha V. To study the effectiveness of self-instructional module on knowledge and selected outcome among women undergoing hysterectomy in a tertiary care hospital in South India. Int J Reprod Contracept Obstet Gynecol. 2017;6:100-5.
13. Shekha. Prevalence, sociodemographic determinants and self-reported reasons for hysterectomy in India. Reproductive Health. 2019;16:11.

14. Uskul. Women's hysterectomy experiences and decision-making. women and health. 2003;38:1.

15. Janda M. Patient-reported experiences after hysterectomy: A cross-sectional study of the views of over 2300 women. J Patient Experience.2019;1-8.

16. Kiyohasa LY. Surgery information reduces anxiety. Brazil. 2004;59(2):151-5.

17. Dennerstein L, Koochaki P, Barton I, Graziottin A. Hypoactive sexual desire disorder in menopausal women: a survey of Western European women. J Sex Med. 2006;3(2):212-22.

18. Kumari VJ, Jaculin. Effectiveness of structured teaching programme on pre and postoperative self care management among women who are undergoing abdominal hysterectomy in selected maternity hospital at Bangalore. Manipal university 2012.

19. Sharmila R. Preoperative teaching about postoperative care among hysterectomy patients. Nightingale Nursing Times. 2012;8(6):62-5.

20. Schoenfelder T, Schaal T, Klewer J, Kugler J. Patient satisfaction and willingness to return to the provider among women undergoing gynaecological surgery. Arch. Gynaecol. Obstet. 2014;290:683-90.

Cite this article as: Jindal M, Naik VR,

Sahasrabhojanee M, Cacodcar J, Kulkarni MS, Naik S. Study on awareness, perceptions, experiences in decision process of hysterectomy in women presenting to Goa Medical College. Int J Reprod Contracept Obstet Gynecol 2021;10:1089-94. 